\title{
A low-cost motion capture system for small-scale wave energy device tank testing
}

\author{
Francesco Paparella \\ Centre for Ocean Energy Research (COER) \\ Maynooth University \\ Co. Kildare, Ireland \\ E-mail: francesco.paparella.2014@mumail.ie
}

\author{
Daniel Toal \\ Mobile and Marine Robotics Research Centre \\ Marine and Renewable Energy Ireland (MaREI) \\ University of Limerick \\ Limerick, Ireland \\ E-mail: daniel.toal@ul.ie
}

\author{
Satja Sivcev \\ Mobile and Marine Robotics Research Centre \\ Marine and Renewable Energy Ireland (MaREI) \\ University of Limerick \\ Limerick, Ireland \\ E-mail: satja.sivcev@ul.ie \\ John V. Ringwood \\ Centre for Ocean Energy Research (COER) \\ Maynooth University \\ Co. Kildare, Ireland \\ E-mail: john.ringwood@nuim.ie
}

The measurement of the motion of a small scale wave energy device during wave tank tests is important for the evaluation of its response to waves and the assessment of power production. Usually, the motion of a small scale wave energy converter is measured using an optical motion tracking system with high precision and sampling rate. However, the cost for an optical motion tracking system can be considerably high and, therefore, the overall cost for tank testing is increased. This paper proposes a low-cost capture system composed of an inertial measurement unit and ultrasound sensors. The measurements from the ultrasound sensors are combined optimally with the measurements from the inertial measurement unit through an extended Kalman filter in order to obtain an accurate estimation of the motion of a wave energy converter.

\section{Introduction}

Wave tank tests are fundamental for the evaluation of the performance of a Wave Energy Converter (WEC). Usually, wave tank tests are carried out on a small WEC prototype, and the results are then scaled for the full scale device. The measurement of the motion of the small scale device, together with measurement of the wave conditions, is important in order to evaluate the response of the device to the action of the waves and assess the mechanical power production.

Usually, the motion of a small scale wave energy converter is tracked using an optical motion capture system which is composed of several cameras positioned around the device [1]. The optical motion capture system can mea- sure the 6 degrees of freedom of a body in a 3D space, i.e. surge, sway, heave, roll, pitch and yaw. Reflective markers are mounted at different points on the body, and the cameras track their motion with high-precision and high sampling rate. In [2], a tank test facility with an optical motion capture system with a position accuracy of $0.9 \mathrm{~mm}$ at $480 \mathrm{~Hz}$ sample rate is presented. In [3], tank tests are carried out with the Qualisys Motion Capture System [4], which is a popular system for motion capture in wave tanks.

The principal drawback of optical motion capture systems is their cost, which can be considerable high and, therefore, the overall cost for wave tank testing is increased. In this paper, a low-cost motion capture system, based on an optimal combination of an Inertial Navigation System (INS) with ultrasound sensors, is proposed. The INS is based on an Inertial Measurement Unit (IMU) which is mounted on the device and measures the accelerations and angular velocities of the device [5]. Given the initial values on position, velocity and orientation of the body with respect to the fixed reference frame, the accelerations and angular velocities provided by the IMU can be integrated in order to determine position, velocity and orientation at any time instant [6]. However, due to low-frequency noise and bias in the IMU, the integration process can lead to drift in the position and orientation with an unbounded error that grows with time [7], [8].

The ultrasonic measurement system is composed of a set of receivers placed around the device and a transmitter which is mounted on the same point of the device where the IMU is placed. The ultrasound sensors measure the absolute position of the device with respect to a fixed reference frame. For the study in this paper, the ultrasound sensor provides 

other hand, the position provided by the ultrasound sensor is characterized by long-term stability, as opposed to the position obtained through the integration of the IMU outputs.

Therefore, the INS and ultrasound sensor have complementary strengths, which make the integration of the two system desirable [9]. In [10], a motion capture system based on an INS, aided by ultrasound sensors, for an augmented reality system is presented. For vehicle applications, the INS is usually aided by radio sensors [11], the Global Position System (GPS) [12], [13] or ground-based radar [14].

In the available literature, several approaches have been developed for aiding the INS by an external global measurement system: uncoupled, loosely coupled, tightly coupled and ultra-tightly coupled systems [14], [15], [16]. In this paper, a loosely coupled ultrasound sensor aided INS with an Extended Kalman Filter (EKF) is proposed [17]. In a loosely coupled method, the difference between the estimated position given by the INS and ultrasound sensors is used to drive an EKF, whose model represents the propagation of the errors of the INS with time. Then, the estimated errors, computed by the EKF, are used to correct the position, velocity and orientation provided by the INS.

The remainder of the paper is organized as follows: in Section 2, the wave tank and the motion sensors are presented while, in Section 3, a fusion algorithm, based on the EKF, is designed in order to optimally combine the data provided by the INS and ultrasound sensors while, in Section 4, the protocol and results on the validation of the fusion algorithm is presented. Finally, overall conclusions are drawn in Section 5.

\section{Proposed wave tank and sensor suite}

The wave tank test system is composed of a wave tank, wave makers, wave gauges and a motion capture system. The wave tank considered in this paper is produced by OMEY labs [18], and is shown in Figure 1. The wave tank is 10 $m$ long, $2.5 m$ wide and $1.3 m$ deep, and is equipped with a bottom-hinged paddle wave maker which can create planar regular and irregular waves, and a parabolic passive beach, for the absorption of the waves. The periods of the regular waves that can be created by the wave maker range from 0.5 $s$ to $3 s$.

The motion capture system of the WEC, which is the focus of this paper, is composed of an inertial measurement unit (IMU) and an ultrasound measurement system from Hexamite [19]. The sampling frequency of the ultrasound system is $15 \mathrm{~Hz}$. The ultrasound system is composed of 4 ultrasound receivers positioned around the device and one ultrasound transmitter which is attached to the top of the WEC, as shown in Figure 2. The weight of the ultrasound transmitter is just $24 \mathrm{~g}$, and is $45 \mathrm{~mm}$ wide, $45 \mathrm{~mm}$ long and $18 \mathrm{~mm}$ high. The weight and dimensions of the transmitter are therefore negligible compared to the weight and size of a small scale WEC, which can weigh up to $10 \mathrm{~kg}$ and be more than $0.5 \mathrm{~m}$ in length and width. As shown in Figure 2, the receivers are positioned on a plane $2.5 \mathrm{~m}$ wide and $2 \mathrm{~m}$ long

at $2.0 \mathrm{~m}$ above the equilibrium free surface elevation. While the width of the plane of the receivers is determined by the width of the wave tank, it was found experimentally that the distance from the free surface elevation has to be above 1.5 $m$ so that each receiver can detect the sound wave emitted by the transmitter. The length of the plane was appropriately chosen in order to cover the range of motion of the WEC.

The ultrasound measures the absolute position of the WEC with respect to a fixed reference frame $X_{g}, Y_{g}, Z_{g}$ located at the position of one of the receivers. Given the speed of sound, the distance of the WEC from each receiver is obtained by measuring the travel time needed by the ultrasonic signal emitted by the transmitter to reach each receiver. As shown in Figure 3, the global coordinates $x_{T}^{g}, y_{T}^{g}, z_{T}^{g}$ of the WEC can be computed, through trilateration, as follows:

$$
\begin{aligned}
x_{T}^{g} & =\frac{r_{1}^{2}-r_{2}^{2}+d^{2}}{2 d} \\
y_{T}^{g} & =\frac{r_{1}^{2}-r_{3}^{2}+B^{2}+C^{2}}{2 C}-\frac{B}{C} x^{g} \\
z_{T}^{g} & = \pm \sqrt{r_{1}^{2}-\left(x^{g}\right)^{2}-\left(y^{g}\right)^{2}}
\end{aligned}
$$

where $r_{1}, r_{2}$ and $r_{3}$ are the distances of the WEC from receivers $R 1, R 2$ and $R 3$, respectively. The parameter $d$ is the distance of receiver $R 2$ from receiver $R 1$ along the $X_{g}$ axis, while the parameters $B$ and $C$ are the coordinates of receiver $R 3$ along the $X_{g}$ and $Y_{g}$ axis, respectively. As shown in Equations (1)-(3), only 3 receivers are needed to calculate the global position of the WEC, with the 4th receiver used for redundancy, in case of failure of one of the other receivers.

The IMU is a LSM6DS0 from STMicroelectronics [20] which is composed of 3 accelerometers and 3 gyroscopes. As shown in Figure 2, the IMU is placed at the same point as the ultrasound transmitter. The accelerometer measures the acceleration along the 3 axis of translation $X_{b}, Y_{b}$ and $Z_{b}$, while the gyroscope measures the angular velocities around the same axes. The sampling frequency of the IMU is $30 \mathrm{~Hz}$.

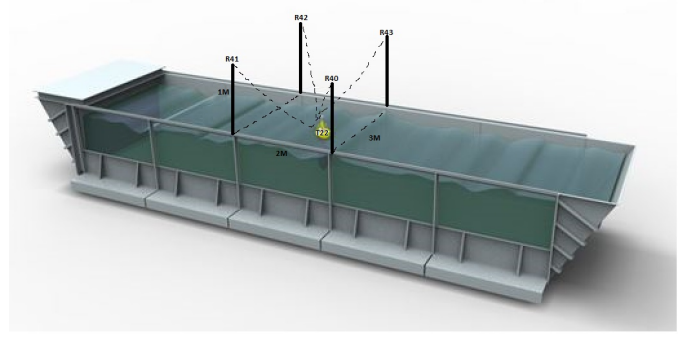

Fig. 1. OMEY labs wave tank with ultrasound receivers placed above the middle section of the tank 
$2 \mathrm{~m}$

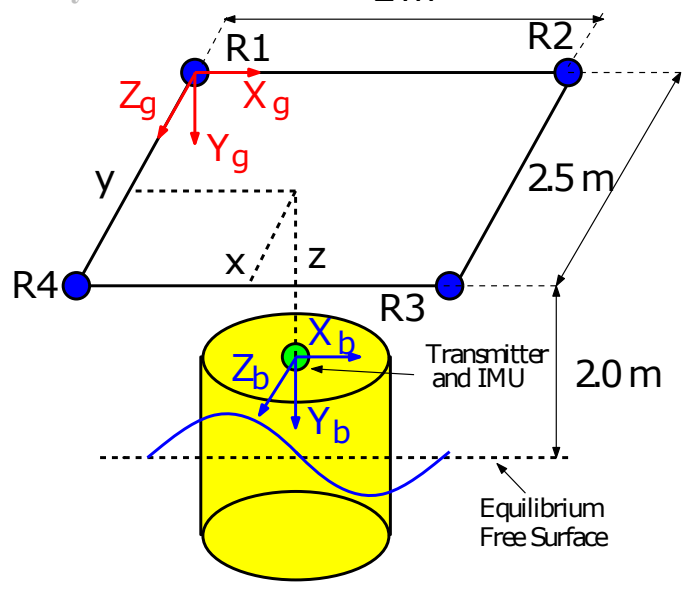

Fig. 2. Set-up ultrasound measurement system and IMU for measurement of the WEC motion

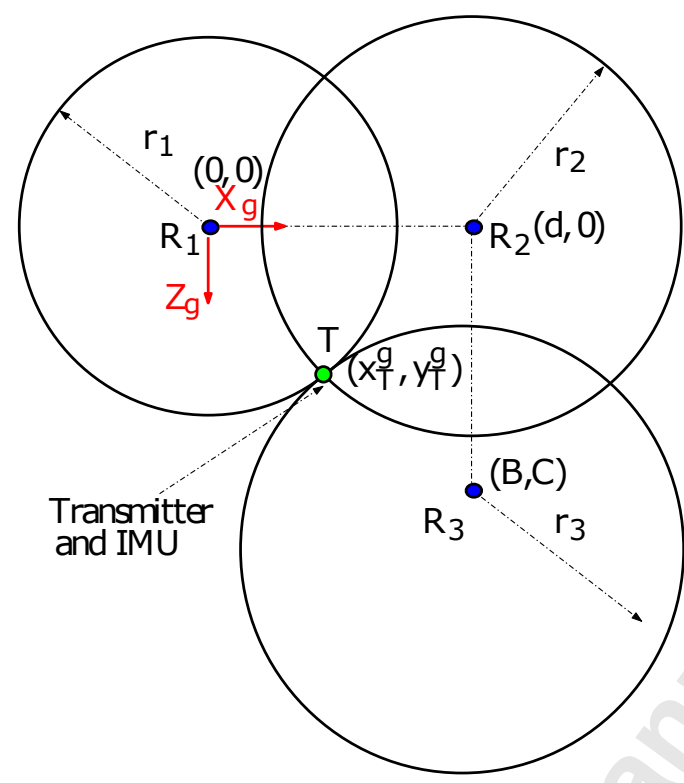

Fig. 3. Intersection of the spheres centered at the location of the receivers $R 1, R 2, R 3$ at the plane $Z_{g}=0$

\section{Sensor processing and optimal combination}

In this section, the measurements from the ultrasound sensors are combined optimally with the measurements from the IMU through an EKF in order to obtain an accurate estimation of the position, velocity and orientation of a WEC. For the position, the EKF estimates are updated using the measurements provided by the ultrasound sensors. Furthermore, since the accelerometer provides a measure of the gravity force vector, the static orientation of the IMU can be detected [21], while the transient orientation of the IMU is provided by the gyroscope. Therefore, the EKF computes the correct position, velocity and orientation from the fusion of the static measurements provided by the ultrasound sensors with the dynamic measurements provided by the IMU.

The INS is described by the inertial navigation equations, which are a set of nonlinear differential equations de- scribing the relationship between the position, velocity and orientation of the WEC, and the outputs from the IMU, which are the accelerations and the angular velocities. The EKF is based on the navigation error equations, which represent a linearized model of the inertial navigation equations. The navigation error equations describe how the errors on the position, velocity and orientation of the WEC relate to the errors on the measured accelerations and angular velocities.

In the reminder of this section, the continuous-time inertial navigation and navigation error equations are obtained. Then, both navigation and navigation error equations are discretized with a zero order hold for the inputs. The discretetime error navigation equations are suitable for the implementation of the EKF with the sampled measurements from both the ultrasound sensors and the IMU. A possible alternative to the discrete-time EKF is a continuous-discrete EKF, based on the continuous-time navigation and navigation error equations, which takes as inputs the measurements provided by the ultrasound sensors and IMU at discrete time instants. However, the software implementation of a continuous-discrete EKF requires an integration scheme, in the time domain, which calculates positions, velocities and orientation at a time instant $t_{k}$ given their values at the time instant $t_{k-1}$. Therefore, the discrete-time EKF is preferred in this paper, since it provides a framework that is suitable for propagating the state variables between discrete time instants, without resorting to an integration scheme in the time domain.

Finally, the EKF is introduced in order to integrate the measurements provided by the ultrasound system with the positions, velocities and orientation computed by the INS.

\subsection{Continuous-time inertial navigation equations}

The continuous-time inertial navigation equations are given as follows [9]:

$$
\begin{aligned}
\dot{\mathbf{p}}^{g} & =\mathbf{v}^{g} \\
\dot{\mathbf{v}}^{g} & =\mathbf{R}_{b}^{g}(\Theta) \mathbf{s}^{b}+\mathbf{g}^{g} \\
\dot{\mathbf{R}}_{b}^{g} & =\mathbf{R}_{b}^{g}(\Theta) \Omega_{g b}^{b}
\end{aligned}
$$

where $\mathbf{p}^{g}$ and $\mathbf{v}^{g}$ are the global position and velocity vector of the WEC, respectively. The vector $\mathbf{g}^{g}$ represents the gravitational force per unit mass, i.e. gravitational acceleration, while $\mathbf{s}^{b}$ represents the inertial force per unit mass, expressed in the body frame, i.e. inertial acceleration. Note that Equations (4a)-(4c) represent a kinematic model, with no forces or torques involved. The matrix $\Omega_{g b}^{b}$ is the skew-symmetric matrix of the angular velocities vector $\omega^{b}=\left[\begin{array}{lll}\omega_{g b_{x}}^{b} & \omega_{g b_{y}}^{b} & \omega_{g b_{z}}^{b}\end{array}\right]^{T}$. The matrix $\mathbf{R}_{b}^{g}(\Theta)$, used for the transformation of coordinates from the body coordinate frame to the global coordinate frame, is a function of the vector of Euler angles $\Theta=[\phi \theta \psi]^{T}$. The state space form of equations (4a)-(4b) can be expressed as: 


$$
\dot{\mathbf{x}}=\mathbf{A d}+\mathbf{B n}
$$

where:

$$
\begin{aligned}
& \mathbf{x}=\left[\begin{array}{ll}
\mathbf{p}^{g, T} & \mathbf{v}^{g, T}
\end{array}\right]^{T} \\
& \mathbf{n}=\mathbf{R}_{b}^{g}(\boldsymbol{\Theta}) \mathbf{s}^{b}+\mathbf{g}^{g} \\
& \mathbf{A}=\left[\begin{array}{ll}
\mathbf{0}_{3} & \mathbf{I}_{3} \\
\mathbf{0}_{3} & \mathbf{0}_{3}
\end{array}\right] \\
& \mathbf{B}=\left[\begin{array}{l}
\mathbf{0}_{3} \\
\mathbf{I}_{3}
\end{array}\right]
\end{aligned}
$$

\subsection{Continuous-time navigation error equations}

The continuous-time navigation error equations are obtained through linearization of the navigation equations around the estimated positions, velocities and orientation computed by the INS. The estimated position, velocity and orientation are given as [9]:

$$
\begin{aligned}
\dot{\hat{\mathbf{p}}}^{g} & =\hat{\mathbf{v}}^{g} \\
\dot{\hat{\mathbf{v}}}^{g} & =\hat{\mathbf{R}}_{b}^{g}(\Theta) \tilde{\mathbf{s}}^{b}+\hat{\mathbf{g}}^{g} \\
\dot{\hat{\mathbf{R}}}_{b}^{g} & =\hat{\mathbf{R}}_{b}^{g}(\Theta) \tilde{\Omega}_{g b}^{b}
\end{aligned}
$$

where the notation $(\hat{\bullet})$ and $(\tilde{*})$ represent estimated and measured quantities, respectively. The estimated rotation matrix $\hat{\mathbf{R}}_{b}^{g}(\Theta)$ is given as follows:

$$
\hat{\mathbf{R}}_{b}^{g}(\Theta)=\left(\mathbf{I}-\Omega_{\varepsilon}\right) \mathbf{R}_{b}^{g}
$$

where $\Omega_{\varepsilon}$ is the skew-symmetric matrix of the elements of the orientation angles error vector $\varepsilon=\left[\begin{array}{lll}\varepsilon_{1} & \varepsilon_{2} & \varepsilon_{3}\end{array}\right]^{T}$. Defining the following errors:

$$
\begin{aligned}
& \delta \mathbf{p}^{g}=\mathbf{p}^{g}-\hat{\mathbf{p}}^{g} \\
& \delta \mathbf{v}^{g}=\mathbf{v}^{g}-\hat{\mathbf{v}}^{g} \\
& \delta \mathbf{s}^{b}=\mathbf{s}^{b}-\tilde{\mathbf{s}}^{b} \\
& \delta \omega_{g b}^{b}=\omega_{g b}^{b}-\tilde{\omega}_{g b}^{b}
\end{aligned}
$$

It is demonstrated in [9] that the continuous-time navigation error equations are given as follows:

$$
\begin{aligned}
\delta \dot{\mathbf{p}}^{g} & =\delta \mathbf{v}^{g} \\
\delta \dot{\mathbf{v}}^{g} & =-\mathbf{S}^{g} \boldsymbol{\varepsilon}+\mathbf{R}_{b}^{g} \delta \mathbf{s}^{b} \\
\dot{\varepsilon} & =\mathbf{R}_{b}^{g} \delta \omega_{g b}^{b}
\end{aligned}
$$

where $\mathbf{S}^{g}$ is the skew-symmetric matrix of the elements of the inertial force per unit mass vector $\mathbf{s}^{g}=\left[\begin{array}{lll}s_{x}^{g} & s_{y}^{g} & s_{z}^{g}\end{array}\right]^{T}$ and is given as follows:

$$
\mathbf{S}^{g}=\left[\begin{array}{ccc}
0 & -s_{z}^{g} & s_{y}^{g} \\
s_{z}^{g} & 0 & -s_{x}^{g} \\
-s_{y}^{g} & s_{x}^{g} & 0
\end{array}\right]
$$

The system of equations in (13) describes how the errors on the positions, velocities and orientations are related to the errors on the measured accelerations $\delta s^{b}$ and angular velocities $\delta \omega_{g b}^{b}$. If the errors $\delta \mathbf{s}^{b}$ and angular velocities $\delta \omega_{g b}^{b}$ are modelled as biases, the state space form of the system of equations in (13) is as follows:

$$
\delta \dot{\mathbf{x}}=\mathbf{F} \delta \mathbf{x}+\mathbf{G u}
$$

where:

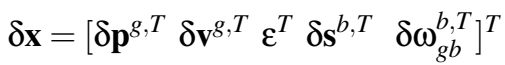

$$
\begin{aligned}
& \mathbf{u}=\left[\begin{array}{ll}
\mathbf{w}_{\text {acc }}^{T} & \mathbf{w}_{\text {gyro }}^{T}
\end{array}\right]^{T} \\
& \mathbf{F}=\left[\begin{array}{ccccc}
\mathbf{0}_{3} & \mathbf{I}_{3} & \mathbf{0}_{3} & \mathbf{0}_{3} & \mathbf{0}_{3} \\
\mathbf{0}_{3} & \mathbf{0}_{3} & -\mathbf{S}^{g} & \mathbf{R}_{b}^{g} & \mathbf{0}_{3} \\
\mathbf{0}_{3} & \mathbf{0}_{3} & \mathbf{0}_{3} & \mathbf{0}_{3} & \mathbf{R}_{b}^{g} \\
\mathbf{0}_{3} & \mathbf{0}_{3} & \mathbf{0}_{3} & \mathbf{0}_{3} & \mathbf{0}_{3} \\
\mathbf{0}_{3} & \mathbf{0}_{3} & \mathbf{0}_{3} & \mathbf{0}_{3} & \mathbf{0}_{3}
\end{array}\right] \\
& \mathbf{G}=\left[\begin{array}{cc}
\mathbf{0}_{3} & \mathbf{0}_{3} \\
\mathbf{R}_{b}^{g} & \mathbf{0}_{3} \\
\mathbf{0}_{3} & \mathbf{R}_{b}^{g} \\
\mathbf{0}_{3} & \mathbf{0}_{3} \\
\mathbf{0}_{3} & \mathbf{0}_{3}
\end{array}\right]
\end{aligned}
$$

with $\mathbf{w}_{\text {acc }}$ and $\mathbf{w}_{\text {gyro }}$ denoting the accelerometer noise and gyroscope noise, respectively. The noises $\mathbf{w}_{\text {acc }}$ and $\mathbf{w}_{\text {gyro }}$ are assumed to be white, with variances $\sigma_{a c c}^{2}$ and $\sigma_{g y r o}^{2}$, respectively. Therefore, the covariance matrix of the measurement noise $\mathbf{u}$ is as follows:

$$
\mathbf{Q}=\left[\begin{array}{cc}
\boldsymbol{\sigma}_{a c c}^{2} \mathbf{I}_{3} & \mathbf{0}_{3} \\
\mathbf{0}_{3} & \sigma_{\text {gyro }}^{2} \mathbf{I}_{3}
\end{array}\right]
$$




\section{Copyright $\mathbf{3 . 3}{ }^{20}$ biscrete time inertial navigation equations}

The continuous-time inertial navigation equations in (5) are discretised to make them suitable for the implementation of the EKF with the sampled measurements from both the ultrasound sensors and IMU. Assuming that the input $\mathbf{n}$ in (5) is constant over a sampling period, the zero-order-hold sampling of the state space in (5) becomes [9]:

$$
\mathbf{x}_{k+1}=\left[\begin{array}{cc}
\mathbf{0}_{3} & T_{s} \mathbf{I}_{3} \\
\mathbf{0}_{3} & \mathbf{0}_{3}
\end{array}\right] \mathbf{x}_{k}+T_{s} \mathbf{n}_{k}
$$

where $T_{S}$ is the sampling period. For the discretisation of the orientation equation in (4c), if the measured angular velocity $\Omega_{g b}^{b}$ is constant over a sampling period, the discrete orientation equation is as follows:

$$
\mathbf{R}_{b, k+1}^{g}=\mathbf{R}_{b, k}^{g}\left(2 \mathbf{I}_{3}+\Omega_{g b, k}^{b} T_{s}\right)\left(2 \mathbf{I}_{3}-\Omega_{g b, k}^{b} T_{s}\right)^{-1}
$$

The vector of Euler angles $\Theta_{k}$, used to represent the orientation of the body coordinate frame, can be computed from $\mathbf{R}_{b, k}[22]$.

\subsection{Discrete time navigation error equations}

The discrete-time inertial navigation error equations are derived from the continuous-time inertial navigation errors equations in (15). If the matrix $\mathbf{F}$ is constant over a sampling period, the discrete-time inertial navigation errors equations are given as follows [9]:

$$
\delta \mathbf{x}_{k+1}=\left(\mathbf{I}+\mathbf{F}\left(k T_{s}\right) T_{s}\right) \delta \mathbf{x}_{k}+\mathbf{u}_{k}
$$

with the covariance matrix of the discrete-time process noise $\mathbf{u}_{k}$ is given as [8]:

$$
\mathbf{Q}_{k}=\mathbf{G}\left(k T_{s}\right) \mathbf{Q G}\left(k T_{s}\right)^{T} T_{s}=\left[\begin{array}{cccr}
\mathbf{0}_{3} & \mathbf{0}_{3} & \mathbf{0}_{3} & \mathbf{0}_{6} \\
\mathbf{0}_{3} & \sigma_{a c c}^{2} \mathbf{I}_{3} & \mathbf{0}_{3} & \mathbf{0}_{6} \\
\mathbf{0}_{3} & \mathbf{0}_{3} & \sigma_{g y r o}^{2} \mathbf{I}_{3} & \mathbf{0}_{6} \\
\mathbf{0}_{6} & \mathbf{0}_{6} & \mathbf{0}_{6} & \mathbf{0}_{6}
\end{array}\right]
$$

\subsection{Extended Kalman Filter}

The EKF integrates the measurements provided by the ultrasounds system with the position, velocities and orientation computed by the INS. Equations (18) and (19) can be written as:

$$
\mathbf{z}_{k+1}=\mathbf{c}\left(\mathbf{z}_{k}, \mathbf{a}_{k}\right)
$$

where $\mathbf{z}_{k}=\left[\mathbf{x}_{k} \Theta_{k}\right]^{T}$ and $\mathbf{a}_{k}=\left[\mathbf{s}_{k}^{b} \omega_{k}^{b}\right]^{T}$. If the measure- ments from the ultrasound system are available, the estimates of the positions, velocities and orientation are given as [9]:

$$
\begin{aligned}
\hat{\mathbf{z}}_{k+1} & =\hat{\mathbf{z}}_{k+1}^{-}+\delta \hat{\mathbf{z}}_{k+1} \\
\hat{\mathbf{a}}_{k+1} & =\tilde{\mathbf{a}}_{k+1}+\delta \hat{\mathbf{a}}_{k+1} \\
\hat{\mathbf{z}}_{k+1}^{-} & =\mathbf{c}\left(\hat{\mathbf{z}}_{k}, \hat{\mathbf{a}}_{k}\right) \\
\delta \hat{\mathbf{z}}_{k+1} & =\left[\delta \hat{\mathbf{x}}_{k+1}\right]_{1, \ldots, 9} \\
\delta \hat{\mathbf{a}}_{k+1} & =\left[\delta \hat{\mathbf{x}}_{k+1}\right]_{10, \ldots, 15} \\
\delta \hat{\mathbf{x}}_{k+1} & =\delta \hat{\mathbf{x}}_{k+1}^{-}+\mathbf{K}_{k+1}\left(\mathbf{y}_{k+1}-\left(\mathbf{H}_{z, k} \hat{\mathbf{z}}_{k+1}^{-}+\mathbf{H}_{x, k} \delta \hat{\mathbf{x}}_{k+1}^{-}\right)\right) \\
\mathbf{K}_{k+1} & =\mathbf{P}_{k+1}^{-} \mathbf{H}_{x, k}^{T}\left(\mathbf{H}_{x, k} \mathbf{P}_{k+1}^{-} \mathbf{H}_{x, k}^{T}+\mathbf{R}_{k}\right)^{-1} \\
\mathbf{P}_{k+1}^{-} & =\left(\mathbf{I}+\mathbf{F}\left(k T_{s}\right) T_{s}\right) \mathbf{P}_{k}\left(\mathbf{I}+\mathbf{F}\left(k T_{s}\right) T_{s}\right)^{T}+\mathbf{Q}_{k} \\
\mathbf{P}_{k+1} & =\mathbf{P}_{k+1}^{-}-\mathbf{K}_{k+1} \mathbf{H}_{x, k} \mathbf{P}_{k+1}^{-} \\
\delta \hat{\mathbf{x}}_{k+1}^{-} & =\left(\mathbf{I}+\mathbf{F}\left(k T_{s}\right) T_{s}\right) \delta \hat{\mathbf{x}}_{k}
\end{aligned}
$$

where $\mathbf{y}_{k+1}$ is the vector of positions provided by the ultrasound system and static orientation measured by the accelerometer, and the matrix $\mathbf{R}_{k}$ is the covariance matrix of the noise on position and static orientation. The matrix $\mathbf{R}_{k}$ is given as follows:

$$
\mathbf{R}_{k}=\left[\begin{array}{cc}
\sigma_{p o s}^{2} \mathbf{I}_{3} & \mathbf{0}_{3} \\
\mathbf{0}_{3} & \sigma_{r o t}^{2} \mathbf{I}_{3}
\end{array}\right]
$$

where $\sigma_{p o s}^{2}$ and $\sigma_{\text {rot }}^{2}$ are the variance of the white noises acting on the position and static rotation measurement, respectively. As shown in Equation (23e), in addition to the estimation of the errors on positions, velocities and orientation angles, the EKF provides an estimate of the biases that affect the measurement of accelerations and angular velocities given by the IMU. The measurement matrixes $\mathbf{H}_{x, k} \in \mathbb{R}^{6,15}$ $\mathbf{H}_{z, k} \in \mathbb{R}^{6,9}$ in Equation (23f) are given as follows:

$$
\begin{aligned}
\mathbf{H}_{x, k} & =\left[\begin{array}{llll}
\mathbf{I}_{3,3} & \mathbf{0}_{3,3} & \mathbf{0}_{3,3} & \mathbf{0}_{3,6} \\
\mathbf{0}_{3,3} & \mathbf{0}_{3,3} & \mathbf{I}_{3,3} & \mathbf{0}_{3,6}
\end{array}\right] \\
\mathbf{H}_{z, k} & =\left[\begin{array}{lll}
\mathbf{I}_{3,3} & \mathbf{0}_{3,3} & \mathbf{0}_{3,3} \\
\mathbf{0}_{3,3} & \mathbf{0}_{3,3} & \mathbf{I}_{3,3}
\end{array}\right]
\end{aligned}
$$

\section{Validation of the sensor system}

In order to validate the motion capture system proposed in Section 2 and the sensor fusion algorithm in Section 3, a series of experimental tests were carried out with a servo controlled six-axis robot arm at the Mobile and Marine Robotics Research Centre at the University of Limerick. The robot is a Stäubli TX Series 60 [23] which has a position repeatability at constant temperature of $\pm 0.02 \mathrm{~mm}$, while the angular resolution for the wrist joint is $0.172^{\circ}$. 


\section{Copyright (4.1 ${ }^{2018 \text { validation protocol }}$}

The setup of the sensors illustrated in Figure 2 was replicated by mounting the ultrasound receivers on a wall along a square of $2.3 \mathrm{~m}$ by $2.3 \mathrm{~m}$, while the IMU, along with the ultrasound transmitter, was mounted on the end effector of the robot which is facing the ultrasound receivers. In Figure 4 , the setup of the IMU and the global coordinate frame of the ultrasound sensors for the tests with the robot is shown. In order to test the static and dynamic performance of the motion capture system, the tests in Table 1 are proposed.

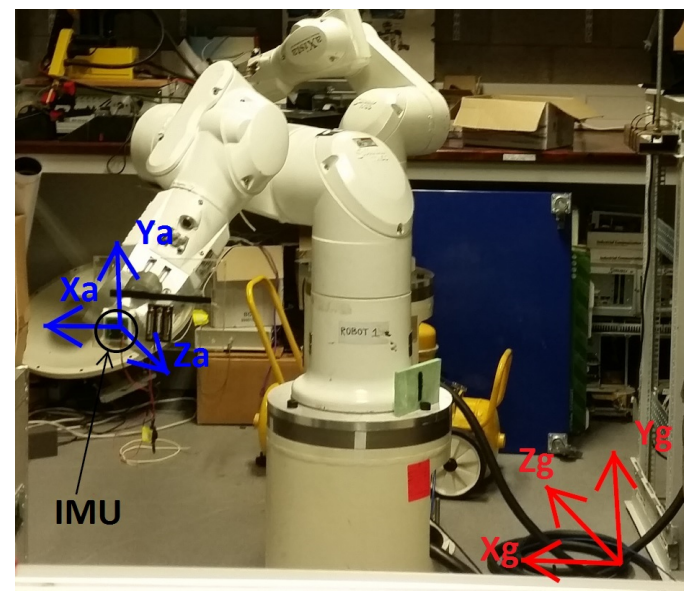

Fig. 4. Set-up of the IMU and ultrasound coordinate frame $X_{g}, Y_{g}, Z_{g}$ for the tests with the Stäubli robot

\subsection{Validation results}

\subsubsection{Static tests}

In this section, the results from the experimental tests with the robot are presented. The measured displacement and errors for $x$-position, $y$-position and $z$-position from the ultrasound sensors for the test SPx, SPy and SPz are shown in Figure 5, 6 and 7, respectively. In order to calibrate the ultrasound sensors along the $x$-axis, $y$-axis and $z$-axis, the measurements are interpolated with a polynomial. The degree of the polynomial is chosen as 5, as it provides a good tradeoff between complexity and interpolation error. As shown in Figure 5, 6 and 7, the interpolant polynomial provides a significantly lower position error than the measurements. The maximum error on $x$-position, $y$-position and $z$-position given by the interpolant polynomial is approximately 0.005 $\mathrm{m}$, which is considerably worse that the accuracy of $0.001 \mathrm{~m}$ stated by the Hexamite company. It is important to highlight that, the EKF does not show any significant improvement of the position accuracy with respect to the ultrasound sensors, since the acceleration measurements provided by the IMU are affected by a considerable bias and, therefore, the position measurement computed by the inertial navigation equations quickly diverges over time.

In order to statically calibrate the accelerometer of the IMU along the $x, y$ and $z$ direction, the tests SRX and SRZ are used. In fact, as the IMU rotates around the $x$-axis and $z$-axis, the components of the gravity vector along the $x$-axis, $y$-axis and $z$-axis of the accelerometer change accordingly. Given a set of different rotations, a calibration procedure for the accelerometers based on the minimization of the squared errors between the applied gravity forces and a model for the accelerometer is proposed in [24]. The cost function to be minimized is as follows:

$$
\min _{\theta} \sum_{k=1}^{N}\left(\left\|\hat{\mathbf{u}}_{k}\right\|^{2}-\| \mathbf{h}\left(\hat{\mathbf{y}}_{k}, \theta \|^{2}\right)^{2}\right.
$$

where $N$ is the number of rotation performed, $\hat{\mathbf{u}}_{k}$ is the applied gravity force vector and $\hat{\mathbf{y}}_{k}$ is the output of the accelerometer. The function $\mathbf{h}\left(\hat{\mathbf{y}}_{k}, \theta\right)$ is given as follows:

$$
\mathbf{h}\left(\hat{\mathbf{y}}_{k}, \theta\right)=\mathbf{T K}^{-1}\left(\hat{\mathbf{y}}_{k}-\mathbf{b}\right)
$$

and:

$$
\begin{aligned}
\mathbf{T} & =\left[\begin{array}{ccc}
1 & -\alpha_{y z} & \alpha_{z y} \\
0 & 1 & -\alpha_{z x} \\
0 & 0 & 1
\end{array}\right] \\
\mathbf{K} & =\operatorname{diag}\left(k_{x}, k_{y}, k_{z}\right) \\
\mathbf{b} & =\left[\beta_{x} \beta_{y} \beta_{z}\right]^{T}
\end{aligned}
$$

where $k_{i}, \beta_{i}$, with $i=x, y, z$, are the unknown scaling and bias parameters of the accelerometer along the $i$ direction. The misalignment angles $\alpha_{i, j}$, with $i=y, z$ and $j=x, y, z$, represents the rotation of the $i$-th accelerometer sensitivity axis around the $j$-th axis of the orthogonal frame.

Regarding the calibration of the gyroscope, the same calibration procedure applied to the accelerometer can be used. The gyroscope sensitivity axes $x, y$ and $z$ are calibrated by using tests SWX, SWY and SWZ, respectively. The cost function to be minimized is shown in equation (27), where $N$ is the number of the constant angular velocities performed. In Table 2, the scale factors and biases of the accelerometer and gyroscope sensitivity axis are shown while, in Table 3, the misalignment angles of the accelerometer and gyroscope sensitivity axes are shown.

For the measurement of the static rotations of the IMU around the $x$-axis, $y$-axis and $z$-axis, two different approaches are considered: integration of the angular velocities provided by the gyroscope and measurement of the applied gravity vector given by the calibrated accelerometer. Note that the integration of the angular velocity diverges over time, since the gyroscope is affected by drift. However, for measurement of the static rotations of the IMU around the $y$-axis, the accelerometer cannot be used, since the gravity vector acts along the $y$-axis and, therefore, no change in the components 


\begin{tabular}{|c|c|c|}
\hline & Static Tests & Dynamic Tests \\
\hline Displacement along $X_{g}, Y_{g}$ and $Z_{g}$ & $\begin{array}{l}\text { SPx, SPy, SPz : range from }-0.4 \mathrm{~m} \\
\text { to } 0.4 \mathrm{~m} \text {, with an increment of } 0.01 \\
\mathrm{~m} .\end{array}$ & $\begin{array}{l}\text { DPx, DPy, DPz: sinusoidal motion } \\
\text { with a peak to peak amplitude of } 0.2 \\
\mathrm{~m} \text { and a range of periods from } 1.25 \\
\mathrm{~s} \text { to } 3 \mathrm{~s} \text {. }\end{array}$ \\
\hline Rotation around $X_{b}, Y_{b}$ and $Z_{b}$ & $\begin{array}{l}\text { SRx, SRy, SRz: range from }-40^{\circ} \text { to } \\
40^{\circ} \text {, with an increment of } 2^{\circ} \text {. } \\
\text { SWx, SWy, SWz: constant rota- } \\
\text { tional velocities with a range from } \\
-229^{\circ} / s \text { to } 229^{\circ} / s \text {, with an incre- } \\
\text { ment of } 10^{\circ} / s \text {. }\end{array}$ & $\begin{array}{l}\text { DRx, DRy, DRz: sinusoidal rota- } \\
\text { tions with amplitude of } 20^{\circ} \text {, with a } \\
\text { range of periods from } 1.25 \mathrm{~s} \text { to } 3 \mathrm{~s} \text {. }\end{array}$ \\
\hline
\end{tabular}

Table 2. Scale factors and biases for accelerometers and gyroscope sensitivity axis

\begin{tabular}{l|l|l|l|l}
\hline & \multicolumn{2}{|c|}{ Accelerometer } & \multicolumn{2}{c}{ Gyroscope } \\
\hline & Scale Factor & Bias & Scale Factor & Bias \\
\hline$X$ & 16.55 & -4.28 & 0.1039 & $7.4 \times 10^{-3}$ \\
\hline$Y$ & 16.65 & 1.35 & 0.1068 & $2.74 \times 10^{-4}$ \\
\hline$Z$ & 16.47 & -8.06 & 0.1152 & $-3.94 \times 10^{-4}$ \\
\hline
\end{tabular}

Table 3. Misalignment angles of accelerometer and gyroscope sensitivity axis

\begin{tabular}{l|l|l}
\hline Angle & Accelerometer & Gyroscope \\
\hline$\alpha_{y z}$ & 0.0039 & -0.0344 \\
\hline$\alpha_{z y}$ & -0.024 & -0.0192 \\
\hline$\alpha_{z x}$ & 0.0128 & -0.0204 \\
\hline
\end{tabular}

of the gravity vector is detected along the $x$-axis and $z$-axis of the accelerometers.

The measured rotations and errors around the $x$-axis and $z$-axis from the accelerometer and gyroscope for the test SRx and SRz are shown in Figure 8 and 10, respectively. The maximum error on the rotation around the $x$-axis is $2^{\circ}$ and $0.9^{\circ}$ for the gyroscope and accelerometer, respectively. The maximum error on the rotation around the $z$-axis is $4^{\circ}$ and $1^{\circ}$ for the gyroscope and accelerometer, respectively.

The measured rotations and errors around the $y$-axis, from the gyroscope $\theta_{\text {meas }}$ for the test SRy, are shown in Figure 9. The maximum error on the rotation around the $y$-axis is $4^{\circ}$. Despite the poor accuracy of the measurement of the rotation angle around the $y$-axis, for a wave tank that only
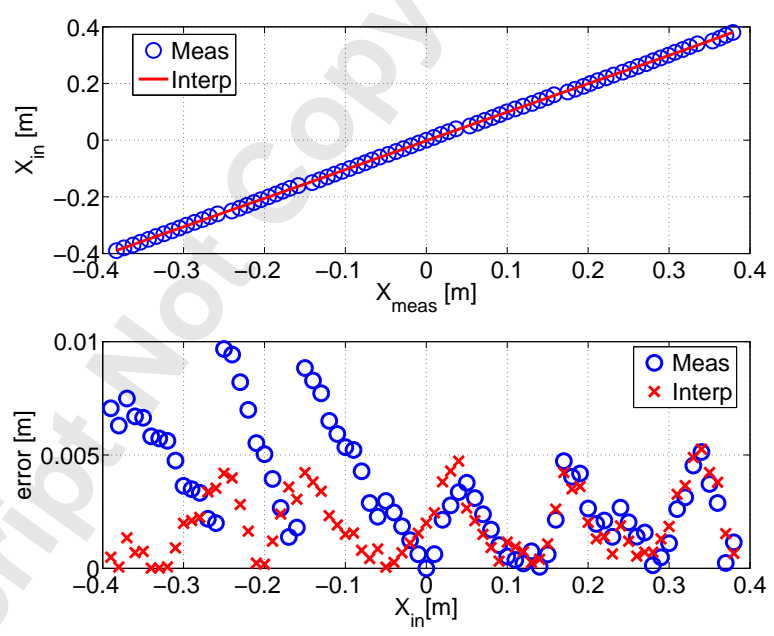

Fig. 5. Measurement of the $x$ positions of the ultrasound transmitter or the test SPX
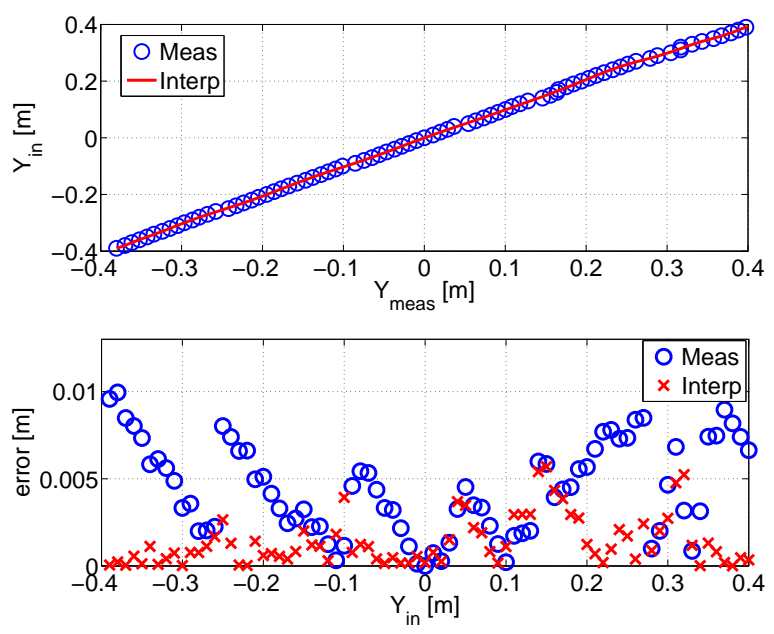

Fig. 6. Measurement of the $y$ positions of the ultrasound transmitter for the test SPy 

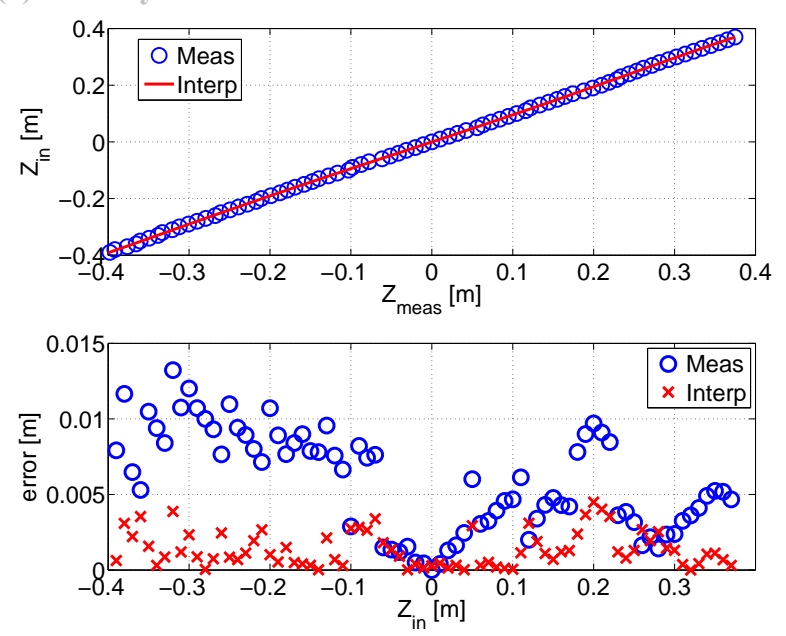

Fig. 7. Measurement of the $z$ positions of the ultrasound transmitter for the test SPz

generates plane waves, the rotation of the device around the axis where the gravity vector is acting upon is negligible. Note that the accuracy of the rotation around the axis along gravity can be improved by using an IMU equipped with a magnetometer, with a small additional cost [21].

In conclusion, for static rotation around the $x$ and $z$ axes, the accelerometer provides a bounded error of $1^{\circ}$, while the gyroscope is affected by a considerable bias, which provides an error of $4^{\circ}$. It is important to highlight that, the EKF does not show any significant improvement of the static rotation accuracy given by accelerometer, since the gyroscope is affected by a considerable bias and, therefore, the rotation measurement computed by the inertial navigation equations quickly diverges over time.
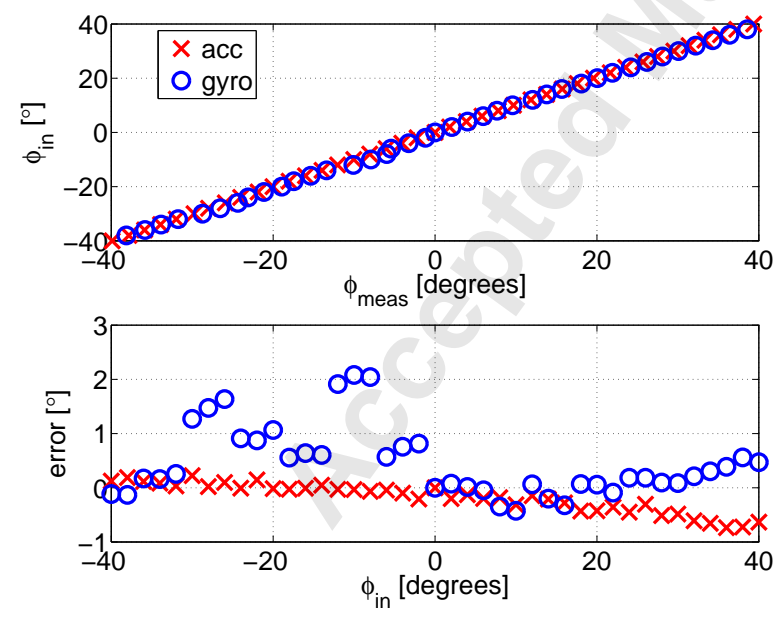

Fig. 8. Measurement of the angle $\phi$ around the $x$-axis of the IMU for the test SRx
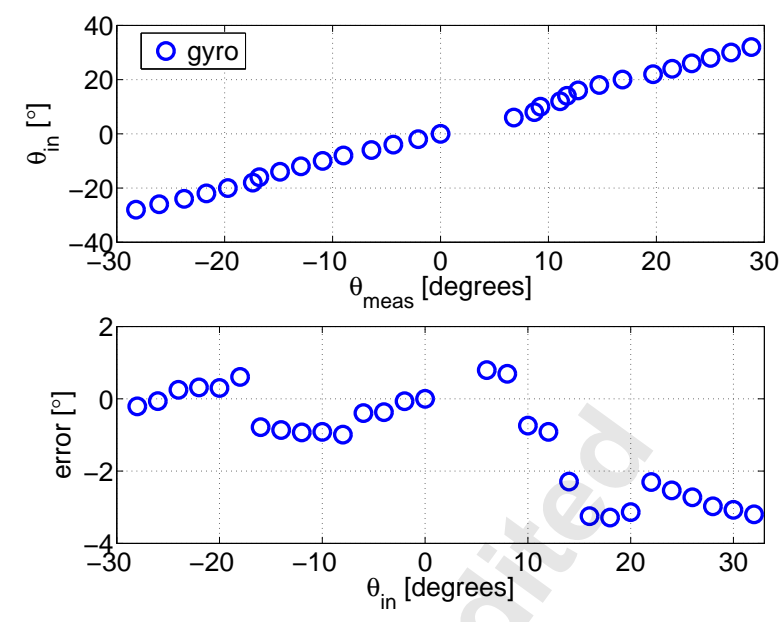

Fig. 9. Measurement of the angle $\theta$ around the $y$-axis of the IMU for the test SRy
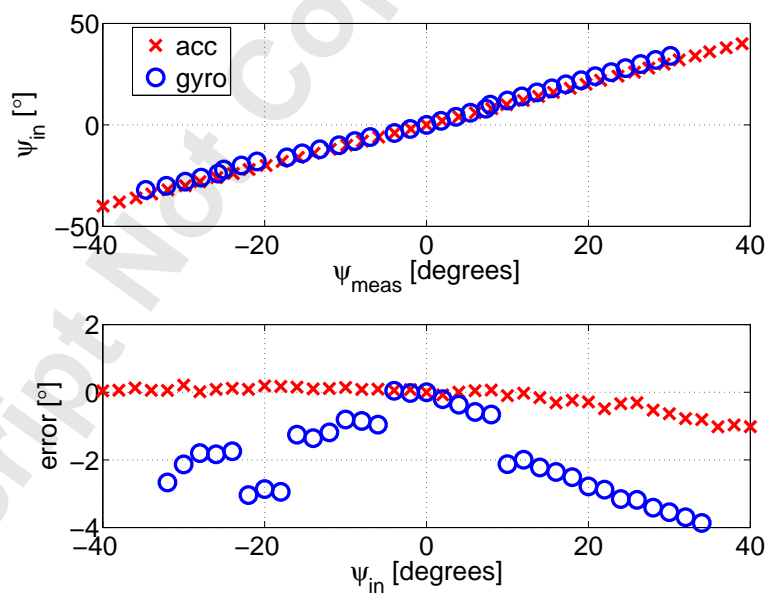

Fig. 10. Measurement of the angle $\psi$ around the $z$-axis of the IMU for the test SRz

\subsubsection{Dynamic tests}

In Table 4 the measured amplitudes and errors for the $x, y$ and $z$ position from the ultrasound transmitter for the tests DPx, DPy and DPz are shown for different periods of sinusoidal trajectories with a reference peak amplitude of 0.2 $m$. Overall, the accuracy on the $x$-position, $y$-position and $z$ position is $0.002 m, 0.005 m$ and $0.005 m$, respectively. Note that, the accuracy along the $x$-axis is better than the accuracies along the $y$ - and $z$-axes across the complete range of tested periods.

In conclusion, for dynamic position measurements, the ultrasound sensors provide an accuracy of $0.005 \mathrm{~m}$ along the $\mathrm{x}, \mathrm{y}$ and $\mathrm{z}$ direction which is the same as the accuracy provided by the static position tests. The EKF does not show any significant improvement of the position accuracy given by the ultrasound sensors. In Table 5 the measured peak-topeak amplitudes and errors of the angle $\phi$ around the $x$-axis and $\psi$ around the $z$-axis from the EKF for the test DRx and $\mathrm{DRz}$ are shown for different periods of sinusoidal trajecto- 
from the ultrasound transmitter for the tests DPx, DPy and DPz

\begin{tabular}{l|l|l|l|l|l|l}
\hline \multirow{2}{*}{$T$} & \multicolumn{2}{|c|}{$X$} & \multicolumn{2}{c|}{$Y$} & \multicolumn{2}{c}{$Z$} \\
\cline { 2 - 7 } & $\begin{array}{l}A_{\text {meas }} \\
{[\mathrm{m}]}\end{array}$ & $\begin{array}{l}e_{\text {meas }} \\
{[\mathrm{m}]}\end{array}$ & $\begin{array}{l}A_{\text {meas }} \\
{[\mathrm{m}]}\end{array}$ & $\begin{array}{l}e_{\text {meas }} \\
{[\mathrm{m}]}\end{array}$ & $\begin{array}{l}A_{\text {meas }} \\
{[\mathrm{m}]}\end{array}$ & $\begin{array}{l}e_{\text {meas }} \\
{[\mathrm{m}]}\end{array}$ \\
\hline 0.8 & 0.195 & 0.005 & 0.2019 & 0.0019 & 0.201 & 0.001 \\
\hline 0.9 & 0.1951 & 0.0048 & 0.1931 & 0.0069 & 0.1995 & 0.0005 \\
\hline 1.25 & 0.1934 & 0.0066 & 0.1975 & 0.0025 & 0.203 & 0.003 \\
\hline 1.6 & 0.197 & 0.0028 & 0.1953 & 0.0047 & 0.2039 & 0.0038 \\
\hline 2 & 0.2001 & 0.0001 & 0.1965 & 0.0035 & 0.1991 & 0.0009 \\
\hline 2.3 & 0.2004 & 0.0004 & 0.2024 & 0.0024 & 0.2042 & 0.0042 \\
\hline 2.65 & 0.2006 & 0.0006 & 0.2018 & 0.0017 & 0.2053 & 0.0053 \\
\hline 3 & 0.2001 & 0.0001 & 0.2042 & 0.0042 & 0.2061 & 0.006 \\
\hline
\end{tabular}

ries with a reference peak-to-peak amplitude of $40^{\circ}$. Note that, for the experimental set-up used in this study, information on the phase was not available, but the tested frequencies are relatively low and no lagging error was observed. Also, at periods lower than $1 \mathrm{~s}$, the translation and rotation of the robot arm is not smooth and, therefore, the uncertainty on the amplitude errors increases. Note that, for both translational and rotational motion, the robot arm can not follow a sinusoidal reference (with a good degree of fidelity) with period less than 0.8 seconds.

As a way of example, in Figure 11, the comparison between the angle $\phi$ computed with the EKF, accelerometers and gyroscope is shown for a sinusoidal angular motion of amplitude $A=20^{\circ}$ and period $T=3.0 \mathrm{~s}$. As Figure 11 shows, for the measurement of the dynamic rotations of the IMU around the $x$-axis, the EKF provides a more accurate estimation of the angles than the angles obtained from the accelerometer or from the integration of the gyroscope angular velocities. Similar considerations can be drawn for the dynamic rotation of the IMU around the $z$-axis shown in Figure 12.

In conclusion, for dynamic rotation measurements, the EKF provides a bounded error of $2^{\circ}$. The gyroscope is affected by a considerable bias and, therefore, the error grows with time. On the other hand, the rotation measurement provided by the accelerometer is not affected by bias but it is noisy.

\section{Conclusions}

This paper proposes a low-cost motion capture system for wave tank tests. The motion capture system is based on two different sensors: ultrasound sensors, which provide position measurements with a low sampling rate but bounded
Table 5. Measured amplitudes and errors of the angle $\phi$ around the $x$-axis and $\psi$ around the $y$-axis of the IMU for the test DRx and DRz

\begin{tabular}{l|l|l|l|l}
\hline \multirow{2}{*}{$T$} & \multicolumn{2}{|c|}{$\phi$} & \multicolumn{2}{c}{$\psi$} \\
\cline { 2 - 5 } & $\begin{array}{l}A_{\text {meas }} \\
{\left[{ }^{\circ}\right]}\end{array}$ & $\begin{array}{l}e_{\text {meas }} \\
{\left[{ }^{\circ}\right]}\end{array}$ & $\begin{array}{l}A_{\text {meas }} \\
{\left[{ }^{\circ}\right]}\end{array}$ & $\begin{array}{l}e_{\text {meas }} \\
{\left[{ }^{\circ}\right]}\end{array}$ \\
\hline 0.8 & 45.11 & 5.11 & 39.63 & 0.36 \\
\hline 0.9 & 43.5 & 3.5 & 40.14 & 0.14 \\
\hline 1.25 & 44.07 & 4.07 & 40.16 & 0.1619 \\
\hline 1.6 & 41.15 & 1.147 & 39.57 & 0.4274 \\
\hline 2 & 39.4 & 0.6042 & 40.53 & 0.5345 \\
\hline 2.3 & 39.74 & 0.2601 & 41.86 & 1.86 \\
\hline 2.65 & 41.41 & 1.412 & 40.71 & 0.7064 \\
\hline 3 & 41.43 & 1.427 & 41.1 & 1.105 \\
\hline
\end{tabular}

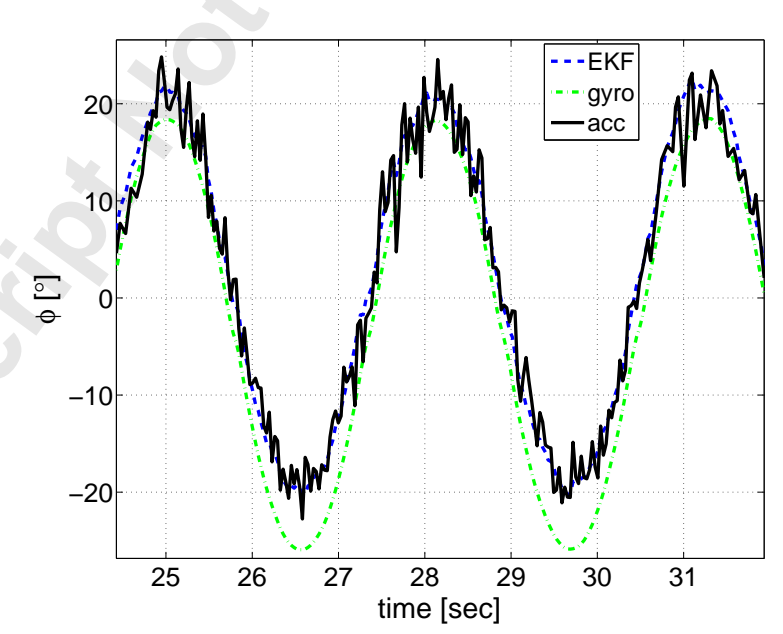

Fig. 11. Comparison between the angle $\phi$ around the $x$-axis of the IMU between computed with the EKF, accelerometer and gyroscope for a period $T=3.0$ and amplitude $A=20^{\circ}$

errors, and an IMU, which provides accelerations and angular velocities with a high sampling rate, but characterized by biases which induces drifts in the integration process used to obtain positions and orientations. Given the complementary strength of the two sensor modalities, a natural choice for the fusion of the information from the two sensors is represented by EKF which estimates positions, velocities and orientation more accurately than the two sensors alone.

The tests performed in this paper show that the ultrasound sensors have a position accuracy of $5 \mathrm{~mm}$ along the $x, y$ and $z$ axes, depending on the range of the position. The position accuracy of the ultrasound sensors is considerably worse than the accuracy stated by the Hexamite company of 


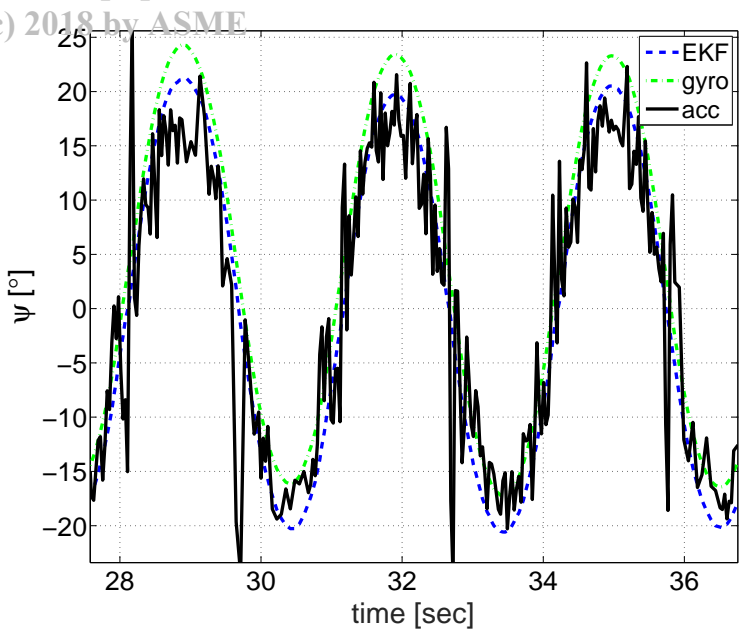

Fig. 12. Comparison between the angle $\psi$ around the $z$-axis of the IMU between computed with the EKF, accelerometer and gyroscope for a period $T=3.0$ and amplitude $A=20^{\circ}$

$1 \mathrm{~mm}$. The EFK does not show any significant improvement of the position accuracy with respect to the ultrasound sensors. An alternative to the ultrasound sensors is a high accuracy optical capture system, which gives an accuracy than is less than $1 \mathrm{~mm}$ but at a more expensive cost [25]. In fact, while the ultrasound measurement system used for the study in this paper costs around $€ 1.5 \mathrm{k}$, the cost for a high accuracy optical capture system can range from $€ 4 \mathrm{k}$ to $€ 10 \mathrm{k}$. A valid low-cost solution for position measurement, with a cost that is comparable to the motion capture system presented in this paper, is the combination of an IMU with 2 High-Definition (HD) webcams. In [26], 2 HD webcams are used to measure the distance of an object from a reference frame, and the results show that the accuracy is less than $2 \mathrm{~mm}$ for the whole range of measurements.

On the other hand, for rotation measurements, the overall accuracy provided by the EKF is good, with bounded errors of $2^{\circ}$ around the $x$ and $y$ axis, depending on the range of the rotation. The rotation computed by the integration of the angular velocity provided by the gyroscope is characterized by an error that grows over time. The accelerometer provides an accurate and stable measure of the static rotation, but the dynamic accuracy is affected by the level of noise in the measurements. The EKF optimally combines the strengths of the gyroscope and accelerometer to compute an accurate and stable rotation both in the static and dynamic scenario.

\section{Acknowledgment}

This paper is based upon works supported by the Science Foundation Ireland under Grant No. 12/RC/2302 for the Marine Renewable Ireland (MaREI) centre.

\section{References}

[1] Marinet, "Tank test related instrumentation and best practice," Marinet, Tech. Rep., 2014.
[2] Rhinefrank, K., Schacher, A., Prudell, J., Stillinger, C., Naviaux, D., Brekken, T., Jouanne, A.V., Newborn, D., Yim, S., and Cox, D., "High resolution wave tank testing of scaled wave energy devices," in Proc. 29th International Conference on Offshore Mechanics and Arctic Engineering (OMAE), 2010.

[3] Bosma, B., Sheng, W., and Thiebaut, F., "Performance assessment of a floating power system for the galway bay wave energy test site," in Proc. International Conference on Ocean Energy (ICOE), 2014.

[4] Qualisys. Available from: http://www.qualisys.com/.

[5] Qin, Z., Baron, L., and Birglen, L., "Robust design of inertial measurement units based on accelerometers," Journal of Dynamic Systems, Measurement, and Control, vol. 131, pp. 031 010-1, 031 010-7, 2009.

[6] Welch, G. and Foxlin, E., "Motion tracking: No silver bullet, but a respectable arsenal," IEEE Computer Graphics and Applications, vol. 22, no. 6, pp. 24-38, 2002.

[7] Cooper, S. and Durrant-Whyte, H., "A Kalman filter model for GPS navigation of land vehicles," in Proc. IEEE/RSJ/GI International Conference on Intelligent Robots and Systems '94. 'Advanced Robotic Systems and the Real World', IROS '94, 1994.

[8] Farrell, J. and Barth, M., The Global Positioning System and Inertial Navigation. McGraw-Hill, New York, 1999.

[9] Skog, I., "A low-cost GPS Aided inertial navigation system for vehicular applications," Master's thesis, Royal Institute of Technology, Stockholm, Sweden, 2005.

[10] Foxlin, E., Harrington, M., and Pfeifer, G., "Constellation" $T M$ : A wide-range wireless motiontracking system for augmented reality and virtual set applications," in Proc. SIGGRAPH 98 Computer Graphics, 1998.

[11] Sillero, J. B., "Sensor fusion methods for indoor navigation using UWB radio aided INS/DR,' Master's thesis, KTH Royal Institute of Technology, 2012.

[12] Farrell, J.A., Tan, H., and Yang, Y., "Carrier phase gpsaided ins-based vehicle lateral control," Journal of Dynamic Systems, Measurement, and Control, vol. 125, pp. 339-353, 2003.

[13] Cho, S. Y., Lee, H. K., and Park, C. G., "Adaptive redundant inertial measurement unit/global positioning system integration filter structure for fault tolerant navigation," Journal of Dynamic Systems, Measurement, and Control, vol. 136, pp. 021 009-1, $021009-$ 10, 2014.

[14] Titterton, D.H. and Weston, J.L., Strapdown Inertial Navigation Technology -2nd Edition. The Institution of Electrical Engineers and The American Institute of Aeronautics and Astronautics, 2004.

[15] Ryan, J. G. and Bevly, D. M., "On the observability of loosely coupled global positioning system/inertial navigation system integrations with five degree of freedom and four degree of freedom inertial measurement units," Journal of Dynamic Systems, Measurement, and 
[16] Johansen, T. A., Hansen, J. M., and Fossen, T. I., "Nonlinear observer for tightly integrated inertial navigation aided by pseudo-range measurements," Journal of Dynamic Systems, Measurement, and Control, vol. 139, pp. 011 007-1, 011 007-10, 2016.

[17] Hieu, L. N. and Nguyen, V.H., "Loosely coupled GPS/INS integration with Kalman filtering for land vehicle applications," in Proc. 2012 International Conference on Control, Automation and Information Sciences (ICCAIS), 1998.

[18] OMEY labs. Available from: http://www.omeylabs.com/home/4584022093.

[19] Hexamite. Available from: http://www.hexamite.com/.

[20] STMicroelectronics Website. Available from http://www.st.com/en/mems-and-sensors/lsm6ds0.

[21] Zhao, H. and Wang, Z., "Motion measurement using inertial sensors, ultrasonic sensors, and magnetometers with Extended Kalman Filter for data fusion," IEEE Sensors Journal, vol. 12, no. 5, 2012.

[22] Slabaugh, G. G., "Computing euler angles from a rotation matrix," City Univ. London, London, Tech. Rep., 1999.

[23] Stäubli Arm - TX series 60 family, Instruction Manual.

[24] Skog, I. and Händel, P., "Calibration of a MEMS inertial measurement unit," in Proc. XVII IMEKOWORLD CONGRESS Metrology for a Sustainable Development, 2006.

[25] Optitrack Website. Available from: http://www.optitrack.com/hardware.

[26] Sophian, A., Sediono, W., Salahudin, M.R., Shamsuli, M.S.M., and Zaaba, D.Q.A., "Evaluation of 3ddistance measurement accuracy of stereo-vision systems," International Journal of Applied Engineering Research, vol. 12, no. 16, pp. 5946-5951, 2017. 